\title{
On the Possibility of Strong Artificial Life
}

\author{
Jianhui Li \\ School of Philosophy, Beijing Normal University, Beijing, China \\ Email: ljh@bnu.edu.cn
}

How to cite this paper: $\mathrm{Li}$, J. H. (2018). On the Possibility of Strong Artificial Life. Open Journal of Philosophy, 8, 495-505. https://doi.org/10.4236/ojpp.2018.85034

Received: October 17, 2018

Accepted: November 9, 2018

Published: November 12, 2018

Copyright $\odot 2018$ by author and Scientific Research Publishing Inc. This work is licensed under the Creative Commons Attribution International License (CC BY 4.0).

http://creativecommons.org/licenses/by/4.0/

\begin{abstract}
One of the central problems in philosophy of artificial life $(\mathrm{AL})$ is whether the artificial life entities we create can be genuine life. Proponents of strong AL believe that the artificial life entities exhibiting characteristics of natural life in a physical or a virtual environment can be real life. Opponents of strong artificial life, however, think that artificial life entities are not real life or just simulation of natural life. The aim of this paper is to demonstrate which view of strong artificial life is valid. The method is to use philosophical theory and logics to analyze the opponents' arguments. The conclusion is that the opponents' arguments for denying strong $\mathrm{AL}$ are insufficient to exclude the possibility of strong AL.
\end{abstract}

\section{Keywords}

Strong Artificial Life, Functionalism, Metabolism, Multiple Realizations, "Shoes/Fly" Fallacy

\section{Introduction}

Artificial life is a new frontier science arising at the end of 1980s. By using computers as a new experimental tool, researchers in this science try to create artificial entities which exhibit characteristics of natural life in computers or in the outside world (Li, 2004; Li \& Zhang, 2006). Some researchers and philosophers of artificial life believe that these man-made systems ultimately can be genuine life. They think that we not only can create living artificial life in outside physical environments, but also create "living digital creatures" in virtual computer environments (Boden, 1996). These artificial creatures not only can reproduce themselves, but they also can mutate, evolve, and even struggle to get resources from their environments for surviving. Creatures that utilize resources more efficiently will have more offspring (Li, Fu, \& Zhang, 2012).

Other researchers and philosophers disagree with these ideas. They hold that 
human beings can never create genuine living artificial life in computers. According to their views, research of artificial life is significant, but the entities artificial lifers (hereafter "A-lifers") create in computers are only simulations of real life. These entities themselves are not really alive (Emmeche, 1994).

The first viewpoint of artificial life, especially the ideas that digital organisms can be real life has already been called strong artificial life, while the second viewpoint, i.e. the idea that artificial life created in computers can't be real life, has been called weak artificial life (Li, 2004).

The main argument for or against strong artificial life is whether or not artificial life in computers meets the standard of life or definition of life (Bedau, 1996; Bedau, 1998; Lange, 1996). In addition to the argument from definition, philosophers also argue from the relation of material and abstract form, the biological meaning of metabolism, flaws of functionalism, and certain reasoning fallacies. This paper first presents both the proponents' and opponents' views of strong artificial life, then offers a critical review of the opponents' four main arguments, and finally states conclusions.

\section{Arguments for Strong Artificial Life}

Strong artificial life has numerous proponents. The most influential researchers in this area are Chris G. Langton (the founder of the artificial life discipline), J. Doyne Farmer (an active proponent of artificial life), Thomas Ray (the creator of the Tierra model), Rodney Brooks (the behavorist robotist), Mark Bedau (the active philosopher of artificial life), and John Casti (the famous mathematician of complexity theory). According to Langton, "The ultimate goal of the study of artificial life would be to create 'life' in some other medium, ideally a virtual medium where the essence of life has been abstracted from the details of its implementation in any particular hardware (Langton, 1986: p. 147)."

He also said: "Artificial Life will force us to rethink what it is to be 'alive.' The fact is, we have no commonly agreed upon definition of the 'living state'. When asked for a definition, biologists will often point to a long list of characteristic behaviors and features shared by most living things (such as the list collated by Mayr) which includes things like self-reproduction, metabolic activity, mortality, complex organization and behavior, etc. However, as most such lists are constituted of strictly behavioral criteria, it is quite possible that we will soon be able to exhibit computer processes that exhibit all of the behaviors on such a list (Langton, 1991: p. 19)."

Following this way of thinking, J. Doyne Farmer and Aletta d'A Belin enumerated the following list of properties as the common characteristics of life (Farmer \& Belin, 1991: p. 818): 1) Life is a pattern in space-time; 2) Living organism has the ability of self-reproduction; 3) Life stores information of self-representation; 4) Life has the ability of metabolism; 5) Living organisms has functional interactions with the environment; 6) The components of living systems depend on one another; 7) Life has the ability of maintaining stability under perturbations; 8) Life has the ability to evolve. 
Farmer holds that this list is far from perfect. Some organisms, for example, viruses, occupy a middle status between living and non-living systems. "Proto-organisms" in some models of origin of life constitute "partially alive" entities as well. Ecosystems and social systems might also qualify as life in term of this list. Therefore, Farmer said, there is no completely clear boundary between life and non-life: "It seems more appropriate to consider life as a continuum property of organizational patterns, with some more or less alive than others (Farmer \& Belin, 1991: p. 819).”

According to this defining list, Farmer tried to argue that computer viruses are a kind of life. He said, "Although computer viruses live in an artificial medium that we cannot directly see, they nonetheless possess most of the properties we have listed as characteristic of life, except possibly the last two. Computer viruses are already more than just a curiosity, and software infected by viruses is becoming increasingly common... Eventually it is likely that a computer virus will be created with a robust capacity to evolve, that will progress far beyond its initial form (Farmer \& Belin, 1991: p. 821)."

Tierra is another kind of model of artificial life that once created a great stir. Are the digital creatures in Tierra really alive? If we follow Farmer's definition, the "creatures" in Tierra are real life, because all of them meet Farmer's list of life properties. Actually, Thomas Ray, the creator of Tierra, went even further. He claimed that the creatures in Tierra are alive simply because life is the system that can reproduce itself and can evolve open-endedly. This is a far simpler definition. Ray claimed that any system that can reproduce itself and can evolve open-endedly is alive (Ray, 1996: p. 112). Not only can the "creatures" in Tierra self-reproduce, but also they can evolve many new astonishing structures. Therefore, Ray affirmed that his Tierra, as a matter of fact, is a living system.

Other models of artificial life, such as Avida (Adami, 1998), "amoebae" (Pargellis, 2001), etc., are also viewed as alive by some researchers of A-life because they all meet the given criteria of the definition of life.

\section{Some Arguments against Strong Artificial Life}

It is interesting to note that the proponents of strong artificial life are mainly biologists and computer specialists. Most of the philosophers have a prudentially criticizing attitude to strong artificial life. There are four main arguments for these philosophers against strong artificial life. I will discuss them as follows:

\subsection{Digital Organisms Are Not Alive}

Some scholars criticize strong artificial life from the relation between matter and form. They believe that the self-reproduction of digital organisms is completely informational, or formal. Unlike organisms in the real world, digital organisms' self-reproduction is not the consumption of matter and growth of matter. Genuine organisms are not merely formal; they also are physical. Or we can say, they should not be only abstract forms, but also be concrete materials. Organ- 
isms which are living independently on earth are composed of cells. Cells can be seen as a self-organizing structure which contains self-description information. It contains not only a lot of concrete biochemical substance, but also a lot of information that describes its own activities. For example, an amoeba is material, but it is also digital. Through the concrete amoebae, its digital "program" interacts with the external world, namely the physical material of the living cell. In a real cell, genetic information is closely related to the working mechanisms that realize the information. That means the instructions or descriptions of a cell behavior completely depend on the working mechanisms of the molecules of the cell that are decoded these instructions or descriptions. Therefore, in the physical world, it is impossible that life can be pure form. Because virtual lives lack material bodies, thus virtual lives are not genuine lives. Von Neumann's idea that it is possible to abstract the logic of life and to realize it in another new medium is hard to apply it. It is possible to realize our formal theory in a medium, but that will not be the real life activities of the primary life system. A closely related saying of this argument is that virtual life is only simulation of life, not real life. For example, Thomas Ray's "Tierra" is only simulation of evolutionary process of life, not real biological life.

\subsection{The Metabolism of Virtual Life Is Not the Same as the Metabolism of Real Life}

Margaret Boden, a philosopher at the University of Sussex, criticizes strong artificial life from the perspective of metabolism. She believes that virtual organisms lack metabolism in the biological sense. Boden sees metabolism as an important criterion for life. She finds that the proponents of strong artificial life seldom emphasize the importance of metabolism in the definition of life. These scholars sometimes see metabolism as an inessential property of life, though it is a universal characteristic of actual life. Or sometimes they say that artificial life models also have metabolism. Boden thinks that the first viewpoint is unacceptable. The second viewpoint seemingly admits that metabolism is required, but the "metabolism" involved here is no longer the real metabolism biological entities have.

So, what is the real meaning of biological metabolism? Before answering this question, Boden differentiates three meanings of metabolism (Boden, 1999): energy dependency, packaged energy dependency, and autonomous use of matter and energy. The first meaning of metabolism is a general energy dependency, i.e. a condition for the existence and persistence of an existence. Yet, Boden holds that this is not metabolism in the biological sense. Mountains and chairs also need energy to maintain their existence, but this is not biological metabolism. There is more to biological metabolism than the mere energy dependence. Biological metabolism not only implicates general energy dependence, but also implicates using, collecting, spending, storing, and budgeting energy.

A second sense of metabolism adds the idea of individual energy packets to mere energy dependency. For example, all living organisms have energy packets 
in their bodies and use these packets to power their life activities. "Every living system has assigned to it, or collects for itself, a finite amount of energy". (Boden, 1999: p. 235) When an individual organism engages in various activities, this energy is spent or deposited. We can see that this second sense of metabolism is stronger than the first one, but Boden believes, it still is not the real biological sense of metabolism. For example, all the autonomous robots have their individual energy packets to support their physical behaviors. They use their stored energy to move around, and they can even recharge their batteries before their energy is used up. Some of the robots even have distinct energy stores devoted to different types of activity. But as we know, the bodies and energy packets of the robots are created by man. Robots do not construct themselves. There are two features of the second definition: "First, it speaks of the creature's 'physical existence', not of the creature's 'body'-nor even of its being a 'unitary' physical system. Second, and crucially, it speaks of that physical existence being taken for grant." (Boden, 1999: p. 236) As we know, an organism's physical existence is an integrated material system and no one takes the existence of a creature's body for granted. "On the contrary, one of the prime puzzles of biology living bodies come into existence, and how they are maintained until the organisms die. We therefore need a third, still stronger, definition of metabolism if we are to capture what biologists normally mean by the term (Boden, 1999: p. 236)".

The third sense of metabolism Boden thinks is "the use, and budgeting, of energy for bodily construction and maintenance, as well as for behavior (Boden 1999: p. 236)". This sense of metabolism does not take the physical existence of the organism for granted, but sees it as constructed in the process of the self-organization of the organism. And unlike inorganic self-organizing systems, the biological meaning of self-organization contains "the autonomous use of matter and energy in building, growing, developing, and maintaining the bodily fabric of a living thing (Boden, 1999: p. 237)". Metabolism needs material substance as a bodily construction matter, and needs energy to organize these material things together as well during the organism's lifetime, forming a being that can persistent even when the outward conditions change. Therefore, the metabolism of real organisms in nature must not only exchange energy with surroundings, but also must budget its inner energy. In this way, excess energy is stored. This can result not only in a saving of energy, but also in the use of that energy when external energy is not sufficient.

Boden believes, if energy dependence is the whole sense of metabolism, then strong artificial life is possible. For strong artificial life is utterly dependent on energy. Without energy, the information process that defines strong artificial life will not be expressed. "Pull the plugs on the computers, stop the electrons inside from jumping, and cyberspace is not merely emptied, but destroyed. Strong A-life, having once existed, would have died (Boden, 1999: p. 234).”

If metabolism is understood in terms of the second meaning, then part of artificial life entities satisfies this condition. For example, both robots and artificial animals have their own specific energy stores, some of which even can abandon 
their current activities in order to recharge their batteries. But virtual artificial life does not satisfy this condition. Virtual organisms exist only in computer memory, manifested to the observer on the VDU-screen. They consist of a particular distribution of electric charges at various (perhaps widely scattered) locations inside the machine. In this sense, then, they may be said to have a physical existence. But that's not to say that they have bodies. Nor is it to say that they store and budget real energy so as to engage in their activities and continue their physical existence. Therefore, they do not satisfy the second definition of metabolism. Thus, virtual life is excluded from real life.

If metabolism is understood in terms of the third definition, then strong artificial life is impossible. First of all, artificial life robots and artificial animals do not satisfy this definition. Though artificial life robots are situated agents that can response to surrounding signal directly, unlike real life, its body structure can't autonomously generate and maintain itself. The third sense of metabolism requires self organization of the system, but present artificial life robots can't meet this. Secondly, virtual artificial life does not conform either. Though virtual artificial life can simulate the third sense of metabolism, being a simulation, it does not possess a genuine bodily self-organization. Therefore, the third sense of metabolism excludes the possibility of all current artificial life models.

But can artificial life be created with true metabolism in the future? Boden thinks that this is an open question. It may be imagined that future artificial life robots might be a self-reproducing material system grounded in a common or an exotic biochemistry. What the exotic biochemistry would look like is not clear. Theoretically it is possible that it is not carbon-based. No matter how these details are spelled out, artifacts built on such biochemistry might fully possess the required life attributes: processing actual metabolism, other than processing digital metabolism in cyberspace. Therefore, this kind of artificial life is possible, but it is actual version of artificial life, other than digital version of artificial life (Boden, 1999).

\subsection{Life May Not Be Multiply Realizable}

The philosopher Elliott Sober at University of Wisconsin-Madison criticizes strong artificial life mainly from its philosophical foundation: functionalism (Sober, 1996). Functionalists believe that living phenomenon might be multiply-realized. Multiple realization means that events defined by their functional properties might have multiple physical realizations. For example, a mouse trap might be composed of iron wire and board, but it might be composed of plastic and spring as well. Each mouse trap is a kind of physical object, but the property of being a mouse trap is not a physical property. Just as there are many ways to build mouse trap, functionalists believe that there are many ways to create life. Life on the Earth happens to be composed of DNA and protein, but life might be composed of other materials, for instance, silicon chips. Perhaps other planets have life, but their compositions might radically differ from terrestrial life. But Sober thinks that how many ways functional property might be realized is an 
empirical question, not a priori one. When functionalists talk of multiple realization, it seems that all properties in the world might be multiply realized. But as a matter of fact, this is not always true. Certain properties might only have one possible realization. Life might be this kind of property. It might be realizable only in carbon-based materials. If life can be realized only in one way, then strong artificial life is impossible.

\subsection{Strong Artificial Life Might Commit a Reasoning Error of "Shoe/Fly Fallacy"}

In addition to the above argument, Sober gives another argument criticizing strong artificial life. Sober points out that functionalists might commit a reasoning error of "shoe/fly fallacy" (Sober, 1996). Functionalists believe that properties of life can be abstracted from the details of their physical realization, but the question is, how many properties can be abstracted as attributes of life? One risk of functionalist theory is that the abstraction of all of these properties is too arbitrary. They often go so far that it is possible for them to confuse biological contents with their mathematical formulation. A real system might in fact not have a mind and it might not be alive, but the confusion we are considering might make us conclude that it has life or mind. Sober gives a simple example to illustrate how this false way of thinking happens. The example is dubbed the "shoe/fly fallacy".

In order to see how the shoe/fly fallacy occurs, Sober uses his familiar biological knowledge. In biology, there is a Hardy-Weinberg law in population genetics. According to the law, the frequency of an allele in a random mating population will not be changed from one generation to the next, if there is no selection, no migration, and the number of male and female individuals is the same. If the frequency of a pair of alleles $\mathrm{A}$ and a is $p$ and $q$ respectively $(p+q=1)$, then the frequency of AA, Aa and aa in the population will be $p^{2}, 2 p q$, and $q^{2}$. If we apply Hardy-Weinberg's law to a population of flies, then we find it reveals an important fact of their reproduction.

Now, suppose shoe makers produces brown and black shoes. Because of an accident, the production line fails to put the shoes in pairs together. Rather, it puts left shoes in one stack and right shoes in the other. The manufacturer then wonders, if he lets a machine randomly pick up one shoe from each stack, then put them together, what will be the results? If $p$ is the frequency of picking black shoes from one stack and $q$ is the frequency of picking brown shoes from the other, then the expected frequency of the three types of pairs is $p^{2}, 2 p q$ and $q^{2}$ respectively. Here we see that the mathematical formulation of shoe paring is the same as the law describing the reproduction of flies. Flies are living, but shoes are not. A machine randomly pairing shoes is not a biological process. Therefore, if we conclude that their subject matters are the same simply because they obey the same laws, we will commit such a reasoning error:

Flies are living things.

Flies are described by law $\mathrm{L}$. 
Shoes are described by law L.

Therefore, shoes are living things.

Sober believes that this reasoning fallacy is a useful tool for preventing us from erroneously judging that an artificial system as alive. To say clearly, though the artificial systems simulated life phenomena by computer follow the same laws as real living things do, they might not the same type of entities.

\section{Critical Thinking on These Arguments}

The above criticisms of strong artificial life seem very reasonable, but actually they have flaws.

\subsection{Virtual Organisms Are Not Just Formal Things}

Some opponents think that virtual organisms are not genuine life because they are only abstract form and lack body. However, as we will see, when we say that the essence of life is form, we do not deny this form depends on a material ground. Actually, artificial lives in computers are not simply forms. They are already the unity of form and matter. Unlike the organisms studied in traditional biology, artificial lives such as computer viruses, Tierra, Avida, and "Amoebae", are not composed of cells, but they are composed of certain electromagnetic states in a computer. We cannot say that these electromagnetic states are not matter. Further, as we already know, the material status of each virtual organism is separate from other matter. Each virtual organism manifests its own individuality. Therefore, these artificial life entities have their own "body", though this body is completely different from the body composed of cells. Some philosophers further say that artificial simulation is only simulation, not real life. Yes, if we see the computer simulations from outside world, they may be only simulations. But how is about to look these simulations from inside of computers? If we see these simulations from inside computers, then these simulations might be real life processes. For example, simulation of replication will be real replication; Simulation of metabolism will be real metabolism; Simulation of growth will be real growth, etc.

\subsection{Strong Metabolism Is Not Necessary}

Boden believes that only the third meaning of metabolism is the relevant biological sense of metabolism. And this kind of metabolism can only be processed by an autopoietic system (a self-maintained and self-created system). An artificially constructed system either is not an autopoietic system, or it only simulates autopoieses. Therefore, up to now, an artificial life system, whether virtual or realistic, does not satisfy the standard definition of life, and is not genuine life. However, Boden admits, it is possible to construct a kind of autopoietic system on some kind of new chemical foundations. This kind of system can process a truly biological sense of metabolism. If this kind of system can be built, then truly living artificial life will be born. But Boden only gives this possibility to 
"realistic artificial life", not to virtual AL. In my opinion, Boden's notion of strong metabolism is an overly rigid criterion for life. For metabolism is only one feature of life; rather, the genuine essence of life is informational flow and genetic heredity. Why must a living thing have genes? From an informational point of view, metabolism is only the means for driving the informational flow and genetic heredity. Therefore, though metabolism is very important to life, we should not see it as the most fundamental property of life. From the time the double helix structure of DNA was discovered, the central study of biology was transferred from metabolism of energy and matter to the flow and heredity of information. If we focus attention on informational flow and heredity, then we can conclude that the role of metabolism is to provide matter and energy for such flow and heredity. For this purpose, the first sense of metabolism can be satisfied. It does not need to satisfy the third definition of metabolism as the only biological sense of life. Certainly, terrestrial life happens to obtain autopoietic metabolism, but now we are go to a new era. In this new century, we want to create life in a computer. In the process of creating of life, our focus should be on the information flow and heredity, rather than on metabolism. Therefore, the first sense of metabolism is enough. Because now we obtain a very good energy source, i.e. electrical energy, we use such kind of convenient energy source naturally in the time of building artificial life. If we require that artificial life must have the same way of utilizing energy source that organisms have, then our effort is to put first thing last. Our mission is to create a dynamic self-replication system of information, rather than how life gathers and uses energy. Therefore, the strong metabolism view of life is misleading, because it requires "life-as-it-could-be" to be completely the same as "life-as-we-now-know-it".

\subsection{Life Can Be Multiple Realization}

Sober's criticism of strong artificial life actually does not exclude the possibility of artificial life. Sober thinks that the problem of multiple realization is an empirical problem. Whether certain properties can be multiply realized or not is purely an empirical question and only empirical evidence can answer it. If life can only be realized in carbon-based molecules, that is, if life can only have one possible realization, then strong artificial life is impossible. But we might postulate in similar manner that if life could be multiply realized, then artificial life is possible. Presently, most people applaud the multiple realization viewpoint of life, thus we can say artificial life is highly possible. Though life is quite complex, many properties of life actually have been shown to be multiply realizable. As a matter of fact, exobiologists have kept studying this problem. They continue probing whether there are other ways of realizing life, though now there still are no exciting results. Artificial life is another new attempt.

\subsection{The "Shoe/Fly" Fallacy Cannot Exclude Artificial Life}

The reasoning of the "shoe/fly fallacy" illustrates that it is useful for preventing 
us from making a simple sort of mistake. However, it should not lead us to deny the possibility of artificial life. The key issue here is what the law of life mentioned in the reasoning is. If the Law is about an arbitrary characteristic of life, then we may assert that the simulation is surely not life. However, if the Law is about an essential characteristic of life, then it is hard to judge whether the simulation is life or not. For example, Sober himself cannot use this reasoning to determine whether computer virus is alive or not. Therefore, the "shoe/fly fallacy" provides us with a good Popperian skeptical instrument, but from this, we cannot conclude that artificial life is impossible. As a matter of fact, Sober himself says, "if a machine can be built that exhibits different biological processes and properties, why we are still interested in saying whether it is alive or not? ... If a machine can perceive, remember, hope, and believe, then what is left of the problem of whether it is thinking? If a machine can absorb energy from its environment, grow, repair physical damage to itself and reproduce, then what is left of the problem of whether it is alive? I cannot see why we will be interested in the universal question after we answer more specific problems (Sober, 1996: p. 376)." Now Sober's view is completely consistent with Langton's view: "We would like to build models that are so life-like that they cease to be models of life and become examples of life themselves (Langton, 1986: p. 147)."

\section{Conclusion}

As we see, one of the central problems in the philosophy of artificial life is whether the artificial life entities we create can be genuine life. The main argument in favor of strong artificial life concerns whether or not artificial life meets the standard of life or definition of life. The proponents of strong artificial life believe that the artificial life entities can be real life because they can be built to meet all the properties of life. Opponents of strong artificial life, however, think that artificial life entities can't meet the standard of life. In this paper, I conclude that all the four arguments for opposing strong artificial life are not sound: virtual lives are not simply formal entities and have material grounds; metabolism is only a means for driving informational flow and genetic heredity and thus the strong metabolism view of life is misleading; the empirical characteristics of multiple realizations do not negate the possibility of multiple realization of life; the "shoe/fly" fallacy cannot exclude artificial life. In a word, present arguments against strong artificial life do not eliminate the possibility of strong artificial life.

\section{Acknowledgements}

This paper is supported by the Planning Office of China Philosophy and Social Science Foundation. The project number is 14ZBD171.

\section{Conflicts of Interest}

The author declares no conflicts of interest regarding the publication of this paper. 


\section{References}

Adami, C. (1998). Introduction to Artificial Life. New York: Springer Verlag. https://doi.org/10.1007/978-1-4612-1650-6

Bedau, M. A. (1996). The Nature of Life. In M. A. Boden (Ed.), The Philosophy of Artificial Life. Oxford: Oxford University Press.

Bedau, M. A. (1998). Four Puzzles about Life. Artificial Life, 4, 125-140. https://doi.org/10.1162/106454698568486

Boden, M. A. (1999). Is Metabolism Necessary? British Journal for the Philosophy of Science, 50, 231-248. https://doi.org/10.1093/bjps/50.2.231

Boden, M. A. (Ed.) (1996). The Philosophy of Artificial Life. Oxford: Oxford University Press.

Emmeche, C. (1994). The Garden in the Machine: The Emerging Science of Artificial Life. Princeton: Princeton University Press.

Farmer, D. F., \& Belin, A. d’A. (1991). Artificial Life: The Coming Evolution. In C. G. Langton, C. Taylor, J. D. Farmer, \& S. Rasmussen (Eds.), Artificial Life II (pp. 815-840). Redwood City, CA: Addison-Wesley.

Lange, M. (1996). Life, Artificial Life, and Scientific Explanation. Philosophy of Science, 63, 225-244. https://doi.org/10.1086/289910

Langton, C. G. (1986). Studying Artificial Life with Cellular Automata. Physica D, 10, 20-149. https://doi.org/10.1016/0167-2789(86)90237-X

Langton, C. G. (1991). Introduction. In C. G. Langton, C. Taylor, J. D. Farmer, \& S. Rasmussen (Eds.), Artificial Life II. Redwood, CA: Addison-Wesley.

Li, J. H. (2004). Towards Computationalism: Philosophy of Artificial Life. Beijing: China Book Press.

Li, J. H., \& Zhang, J. (2006). Digital Genesis: The New Science of Artificial Life. Beijing: Science Press.

Li, J. H., Fu, Z., \& Zhang, J. (2012). Computationalism: A New Kind of World View. Beijing: China Social Science Press.

Pargellis, A. N. (2001). Digital Life Behavior in the Amoeba World. Artificial Life, 7, 63-75. https://doi.org/10.1162/106454601300328025

Ray, T. (1996). An Approach to the Synthesis of Life. In M. A. Boden (Ed.), The Philosophy of Artificial Life. Oxford: Oxford University Press.

Sober, E. (1996). Learning from Functionalism: Prospects for Strong Artificial Life. In M. A. Boden (Ed.), The Philosophy of Artificial Life (pp. 361-378). New York: McGraw-Hill. 